\title{
Characterization of a thyroid hormone-mediated short-loop feedback control of TSH receptor gene in an anaplastic human thyroid cancer cell line
}

\author{
S-T Chen, J-D Lin and K-H Lin ${ }^{1}$ \\ Division of Endocrinology and Metabolism, Chang Gung Memorial Hospital, 5 Fushin St., Kweishan, Taoyuan, Taiwan, Republic of China \\ ${ }^{1}$ Department of Biochemistry, Chang Gung University, Taoyuan, Taiwan, Republic of China \\ (Requests for offprints should be addressed to S-T Chen; Email: stc1105@adm.cgmh.org.tw)
}

\begin{abstract}
The expression of TSH receptor (TSHR) gene is frequently lost in thyroid cancers during the process of dedifferentiation that involves perturbation of several nuclear transcription factors. We have established that thyroid hormone receptor $\beta 1$ (TR $\beta 1)$ is associated with the loss of TSHR gene expression in an anaplastic human thyroid cancer cell line, ARO. To demonstrate that TR $\beta 1$ regulates TSHR gene expression, we performed electrophoresis mobility shift and 3,5,3'-triiodothyronine (T3) transactivation assays. As expected, TR $\beta 1$ bound the synthesized oligomer containing TSHR promoter sequence by heterodimerizing with retinoid $\mathrm{X}$ receptor. When a chimeric reporter pTRCAT5'-146 enclosing the minimal TSHR promoter was applied for T3
\end{abstract}

transactivation assay, two TR $\beta 1$-overexpressing transfectants of ARO cells (ARO1 and ARO2) demonstrated higher basal activity than their parental cells. Consequentially, T3 suppressed the reporter gene activity only in ARO1 and ARO2, but not in ARO cells. A point mutation creating a cAMP response element (CRE) in the reporter pTRCAT5'-146 CRE led to T3-induced suppression of the reporter gene in ARO cells without changing the basal or T3-induced activities in ARO1 and ARO2 cells. We conclude that the regulatory effect of T3 on TSHR gene expression is TR- and promoter DNA sequence-determined.

Journal of Endocrinology (2002) 175, 459-465

\section{Introduction}

As a thyrotropic factor, thyrotropin (TSH) binds to its receptor (TSHR) to regulate the growth and general protein synthesis of the thyroid mainly through the adenylate cyclase (and perhaps also phosphatidyl inositol) pathway (Rapoport \& Spaulding 1996). A sophisticated feedback mechanism induced by TSH has been documented to work through a cAMP-dependent cascade to autoregulate the expression of TSHR gene (Kohn et al. 1995). Through detailed analysis of the promoter region, regulatory elements responding to factors such as cAMP, insulin/insulin-like growth factor-I, thyroid transcription factors (TTFs) and many others, have been determined within the promoter sequence of the TSHR gene (Kohn et al. 1995). In the case of the rat TSHR promoter, a 'minimal region' exhibiting promoter activity was identified between -195 and -39 bp of the $5^{\prime}$ flanking region of the TSHR gene. Among the minimal promoter region, an octameric cAMP response element (CRE)-like sequence, TGAGGTCA, within -139 to $-132 \mathrm{bp}$ of the rat TSHR promoter, has been documented to serve as a constitutive enhancer. Its mutation to a consensus CRE
(TGACGTCA) improved the promoter activity in nonfunctioning rat FRT thyroid cells (Ikuyama et al. 1992). Interestingly enough, an enclosed hexameric sequence, AGGTCA, of the CRE-like structure corresponds to a consensus half site of the thyroid hormone response element (TRE) (Saiardi et al. 1994).

Although thyroid hormone was found to inhibit the regulatory effect of TSH on thyrocytes several decades ago (Cortell \& Rawson. 1944, Takasu et al. 1974, Yu et al. 1976, Akiguchi et al. 1992), the mechanism remained unknown until Saiardi et al. (1994) demonstrated that thyroid hormone receptor/retinoid X receptor (TR/ RXR) complex footprinted the flanking region of the aforementioned hexameric TRE sequence of the rat TSHR promoter. The mechanism by which 3,5,3'triiodothyronine (T3) regulates gene expression has now been elucidated. In short, T3 exerts a dual regulatory function in target gene control; that is, while many genes are activated by T3, a considerable numbers of genes are down-regulated by the same hormone. It is now known that such divergent regulatory effects of $\mathrm{T} 3$ are determined by alignments of a specified DNA motif termed TRE that is usually identified within the promoter region of the 
target gene. As can be demonstrated by functional studies such as nuclear run-on, footprinting and gel shift assays, TRE structures recognized as palindromic, inverted palindromic, and direct repeat spaced with four nucleotides are coupled to positive transactivation of the target gene, and are preferentially bound with the TR/RXR heterodimer (Cheng 1995, Williams \& Brent 1995). In contrast to the positive TRE, the findings of a single (Chatterjee et al. 1989), two (Wood et al. 1989) or more (Harding \& Duester 1992) overlapping putative TR biding sites of the deduced TRE documented from genes down-regulated by T3 made it difficult to draw a consensus structure of the negative TRE (nTRE) (Williams \& Brent 1995). In addition, many nuclear cofactors categorized as corepressors and coactivators participate in target gene regulation. In the absence of T3, the unliganded TR (TR/RXR) intertwines with corepressors to repress (or activate) the basal activity of the downstream gene. After T3 binding, a conformation change induces the release of corepressors and consequentially leaves a space for coactivators to bind to the complex and up- (or down-) regulate gene expression (Koenig 1998).

The absence of TSHR gene expression in neoplastic thyroid cells is not an infrequent feature (Berlingieri et al. 1990, Brabant et al. 1991). This feature was attributed to the loss of TTFs (Civitareale et al. 1993, Schuppert et al. 1996), tumor suppressor gene $p 53$ (Moretti et al. 1997) and TR (Bronnegard et al. 1994) in thyroid cells. By using a TR $\beta 1$ - and TSHR-deficient human anaplastic thyroid cancer cell line, ARO, we found that TSHR gene reappeared after TR $\beta 1$ gene became overexpressed in the cell line (Chen et al. 2000a).

Despite TR $\alpha 1 / \mathrm{RXR}$ heterodimer protecting the rat TSHR minimal promoter from DNase 1 digestion (Saiardi et al. 1994), direct evidence illustrating the binding of TR to the human TSHR promoter is lacking. Based on the finding that the minimal promoter regions of the rat and human TSHR genes are highly homologous (Kohn et al. 1995), we have tried to verify a direct interaction between TR and the human TSHR promoter by carrying out an electrophoresis mobility shift assay (EMSA). Furthermore, to clarify the regulatory effect of T3 on TSHR gene expression, we investigated the transactivating activity of T3 in ARO cells with or without TR $\beta 1$ overexpression. Finally, to determine whether T3 mutually affects the overlapping CRE-like structure, we compared the T3 transactivating activities between reporter genes that contained the minimal rat TSHR promoter with a native (pTRCAT5'-146) and a consensus CRE (pTRCAT5'146 CRE) sequence (Ikuyama et al. 1992).

\section{Materials and Methods}

Cell lines

A TR-deficient, poorly differentiated human thyroid cancer cell line, ARO, was kindly provided by Dr G J F
Juillard (UCLA, Los Angeles, CA, USA). By stable transfection with a human TR $\beta 1$ expression vector pcDNA3·1-51, two lines of TR $\beta 1$-overexpressing ARO transfectants ARO1 and ARO2 were established as previously described (Chen et al. 2000a). Cells were grown in RPMI medium (Invitrogen, Carlsbad, CA, USA) supplemented with $5 \%$ fetal calf serum (FCS), $2 \mathrm{mM}$ L-glutamine (Sigma, St Louis, MO, USA), $100 \mathrm{U} / \mathrm{ml}$ penicillin (Sigma), $0 \cdot 1 \mathrm{mg} / \mathrm{ml}$ streptomycin (Sigma), with or without $400 \mu \mathrm{g} / \mathrm{ml}$ geneticin (G418; Invitrogen).

\section{Plasmids}

Expression vectors containing full-length $\mathrm{cDNA}$ sequences of wild type human TR $\alpha 1$ (pcDNA3.1-61), TR $\beta 1$ (pcDNA3·1-51) and RXR $\alpha$ were kindly provided by $\mathrm{Dr}$ $S$ Y Cheng (National Institutes of Health, Bethesda, MD, USA). A chimeric reporter gene pTRCAT5'-146 enclosing the $-146 /-9$ nucleotide sequence upstream to the transcription start site of rat TSHR gene and its mutant pTRCAT5'-146 CRE were generous gifts from Dr H Shimura (Yamanashi Medical University, Japan). The pTRCAT5'-146 reporter contained an octameric CRElike sequence, TGAGGTCA, in which a hexameric sequence (AGGTCA) of the known TRE half site is documented among nucleotides $-137 /-132$. The mutant pTRCAT5'-146 CRE was reconstructed by a ${ }^{-136} \mathrm{G} / \mathrm{C}$ substitution to create a CRE motif (TGAC GTCA) (Ikuyama et al. 1992).

\section{In vitro transcription/translation}

TR $\alpha 1, T R \beta 1$ and $\mathrm{RXR} \alpha$ proteins were in vitro translated with a TNT Rabbit Reticulocyte Lysate Kit (Promega, Madison, WI, USA) according to the manufacturer's instructions.

\section{EMSA}

The oligomer tshrp (identical to the $-11 /+44 \mathrm{bp}$ fragment of human TSHR promoter and corresponding to the $-166 /-112 \mathrm{bp}$ fragment of rat TSHR promoter (Fig. 1)) was synthesized. The in vitro translated products of human TR $\alpha 1 / \beta 1$ protein, either alone or in combination with $\operatorname{RXR} \alpha$ protein, were premixed with or without TR-specific monoclonal antibody (mAb) C4 (Santa Cruz Biotechnology, Santa Cruz, CA, USA) at room temperature for $30 \mathrm{~min}$, followed by incubation in buffer containing poly-dI/dC (Sigma) and $10^{5}$ c.p.m./reaction $\left[\alpha_{-}{ }^{32} \mathrm{P}\right] \mathrm{dCTP}$ (Amersham Pharmacia Biotech Inc., Piscataway, NJ, USA) end-labeled oligomer at $25^{\circ} \mathrm{C}$ for a further $15 \mathrm{~min}$. During the incubation, non-isotopelabeled specific or non-specific competitors were added to compete for DNA binding. After SDS-PAGE (Sigma), the gel was dried and autoradiographed overnight. In addition 


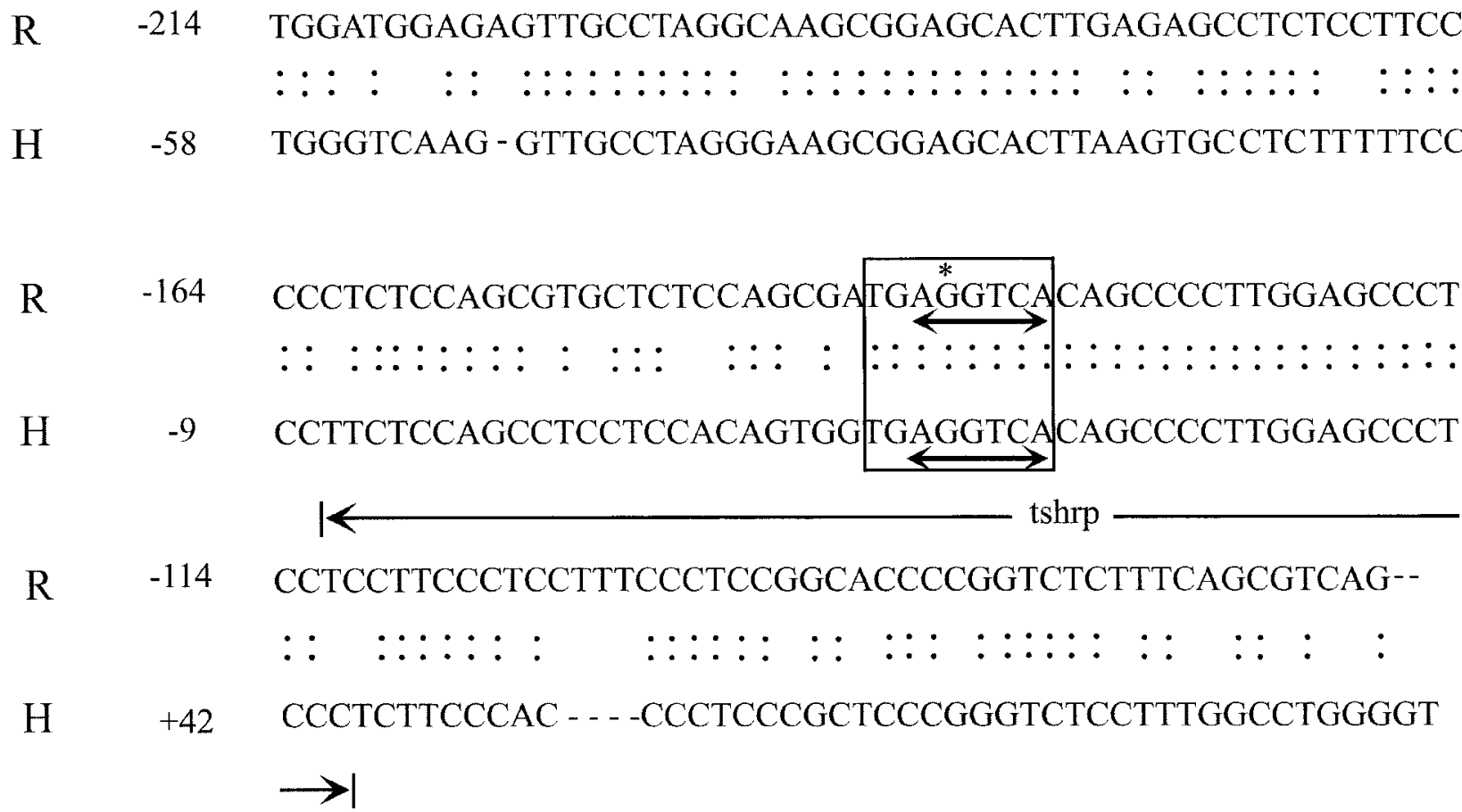

Figure 1 Schematic illustration of the flanking region of a hexameric (AGGTCA, underlined with bold arrowed lines) TRE half site determined from the TSHR promoter. An octameric structure (boxed area) resembling the natural CRE except for a G instead of a C on the fourth nucleotide $\left({ }^{*}\right)$ was found overlapping the TRE sequence. A high degree of homology was found in both of the TSHR promoter fragments illustrated. The synthesized oligomer tshrp (thin arrowed line) was identical to the $-11 \sim+44$ bp fragment of the human (H) TSHR promoter and corresponded to the $-166 \sim-112$ bp fragment of the rat (R) TSHR promoter.

to tshrp, the oligomer F2 that contained an inverted repeat TRE sequence (Lin et al. 2001) served as control.

Transient transfection and chloramphenicol acetyl transferase (CAT) assay

After incubation with serum free medium, $1 \times 10^{6}$ ARO, ARO1 and ARO2 cells were transfected with aliquots of DNA/liposome mixtures containing $1 \mu \mathrm{g}$ pTRCAT5'146, pTRCAT5'-146 CRE or a promoterless CAT plasmid, p0 CAT (Chen et al. 2000b), and $5 \times(\mathrm{v} / \mathrm{v})$ lipofectAMINE (Invitrogen) respectively. After $5 \mathrm{~h}$ incubation, the reaction was stopped by 10\% T3-depleted (Td) FCS and subsequently cultured overnight. Cells were then moved to conditioning media that contained 0 (Td), 1, 10 or $100 \mathrm{nM} \mathrm{T3}$ on the next morning and incubated for a further $48 \mathrm{~h}$. At the end of the incubation period, cells were harvested and cellular proteins were obtained by repetitive freezing and thawing. Aliquots of $100 \mu \mathrm{g}$ protein obtained from each culture condition were incubated overnight with $2 \mu \mathrm{l}\left[1-{ }^{14} \mathrm{C}\right]$ acetyl-CoA (Amersham) and $25 \mu \mathrm{l} 10 \mathrm{mM}$ chloramphenicol (Sigma). CAT activities were assayed with a liquid scintillator. The transfection efficiency was normalized to the protein concentration of the lysates.

\section{Statistics}

Raw data were collected in more than three consecutive experiments and are presented as means \pm S.E.M. A difference was considered significant if the $P$ value was $<0 \cdot 05$ by paired Student's $t$-test.

\section{Results}

TR retarded the mobility of the oligomer tshrp only in complex with RXRa

The oligomer tshrp containing the flanking sequences of the consensus TRE half site enclosed in human TSHR promoter (Fig. 1) was tested for TR binding. When TR $\beta 1$ and TR $\alpha 1$ were applied, neither protein bound the oligomer; nevertheless, co-addition of RXR $\alpha$ enabled TR $\alpha 1$ and, to a lesser extent, TR $\beta 1$ to retard the mobility of the oligomer (Fig. 2a, lanes 5, 14). Furthermore, mAb C4 supershifted the retarded TR/RXR/tshrp complexes (lanes 7, 16). As a positive control, the oligomer F2 that 


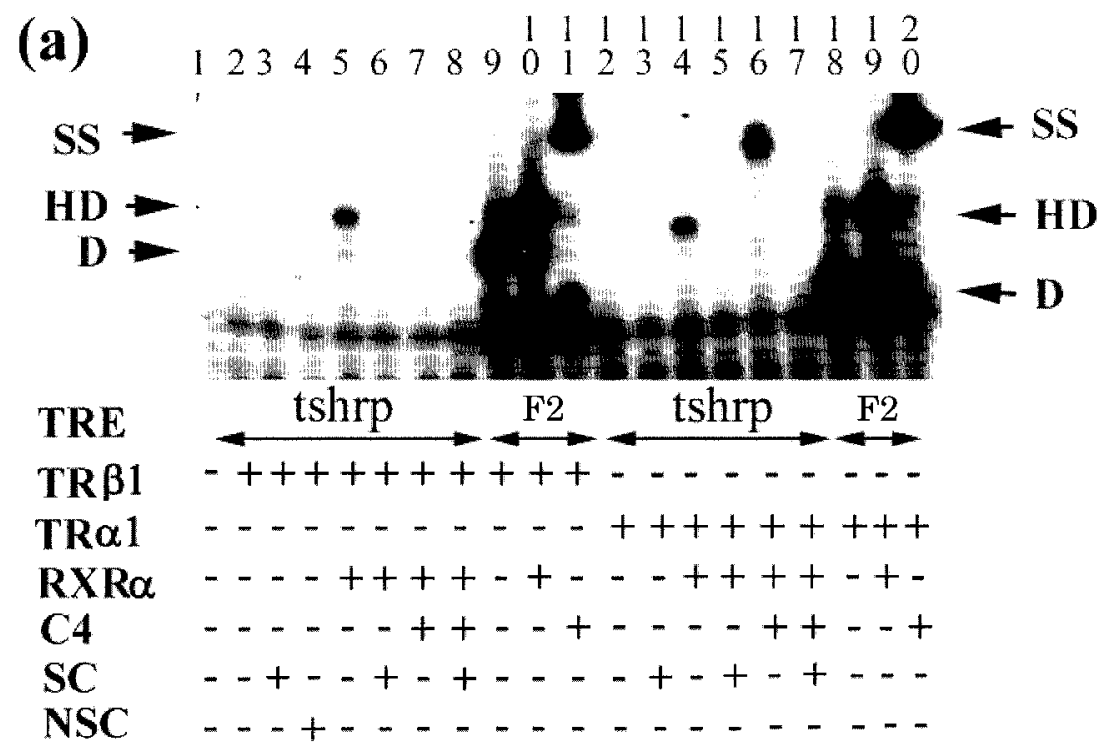

(b) $\quad$\begin{tabular}{lllllllllllllllllll}
1 & 2 & 3 & 4 & 5 & 6 & 7 & 8 & 1 & 1 & 1 & 1 & 1 & 1 & 1 & 1 & 1 \\
\hline & 1 & 1 & 2 & 3 & 4 & 5 & 6 & 7 & 8
\end{tabular}

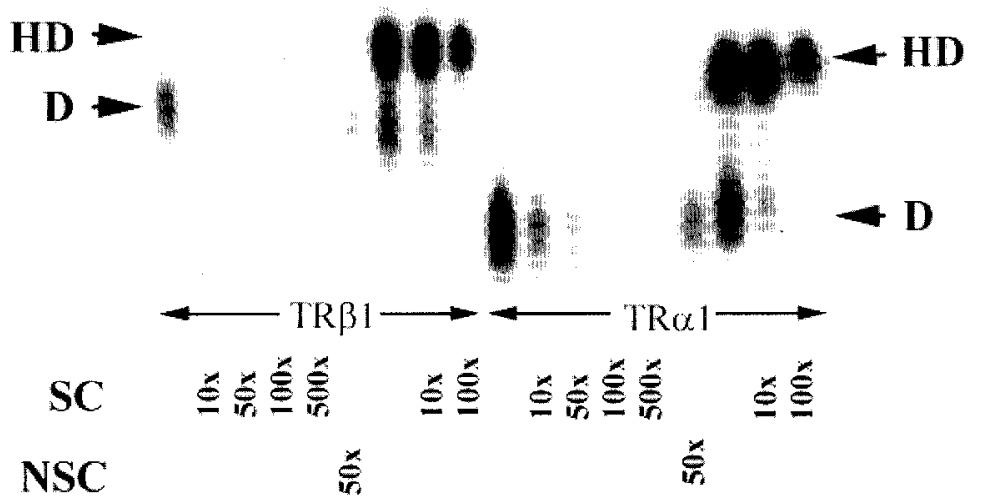

Figure 2 EMSA illustrating TR/RXR proteins retarded tshrp and F2 oligomers. (a) The F2 oligomer was retarded by both TR $\beta 1(\alpha 1)$ homodimers (D; lanes 9, 18) and TR/RXR $\alpha$ heterodimers (HD; lanes 10, 19), which were then supershifted (SS) by TR-specific mAb C4 (lanes 11 and 20). In contrast, TR $\beta 1(\alpha 1)$ alone did not bind to the tshrp oligomer, whereas tshrp was bound by TR/RXR $\alpha$ HD (lanes 5,14 ) and was SS by C4 (lanes 7,16 ). The autoradiographed bands for HD and SS were competed (lanes 6, 8, 15 and 17) when $50 \times$ molar ratio of non-isotope-labeled tshrp (SC) was applied. (b) Bands D represent $\operatorname{TR} \beta 1(\alpha 1)$ homodimer binding to the F2 oligomer (lanes $1-6$ and $10-15$ respectively), which was competed by $10 \times, 50 \times, 100 \times$ and $500 \times$ molar ratios of non-isotope-labeled tshrp (SC), but not competed by a $50 \times$ molar ratio randomized oligomer (NSC, lanes 6 , 15). Similar results were shown in lanes $7-9$ and $16-18$ when $T R / R X R \alpha$ HD was applied.

contained an inverted repeat TRE bound TR homodimers (Fig. 2a, lanes 9, 18) as well as TR/RXR heterodimers (lanes 10, 19). When a non-isotope-labeled tshrp was co-incubated with F2, it competed for the binding of TR homodimers, and TR/RXR $\alpha$ heterodimers to F2 in a molar ratio-dependent manner (Fig. 2b).
T3 suppressed the TSHR reporter gene activity in a TR-dependent manner

Under T3-depleted conditions, the reporter pTRCAT5'146 showed higher basal activities in the TR $\beta 1$ overexpressing (ARO1 and ARO2) cells than in the 
parental cells (Fig. 3a). Addition of T3, even up to $100 \mathrm{nM}$, could not suppress the basal activity of the reporter in ARO cells. Nonetheless, T3 (as little as $1 \mathrm{nM}$ )
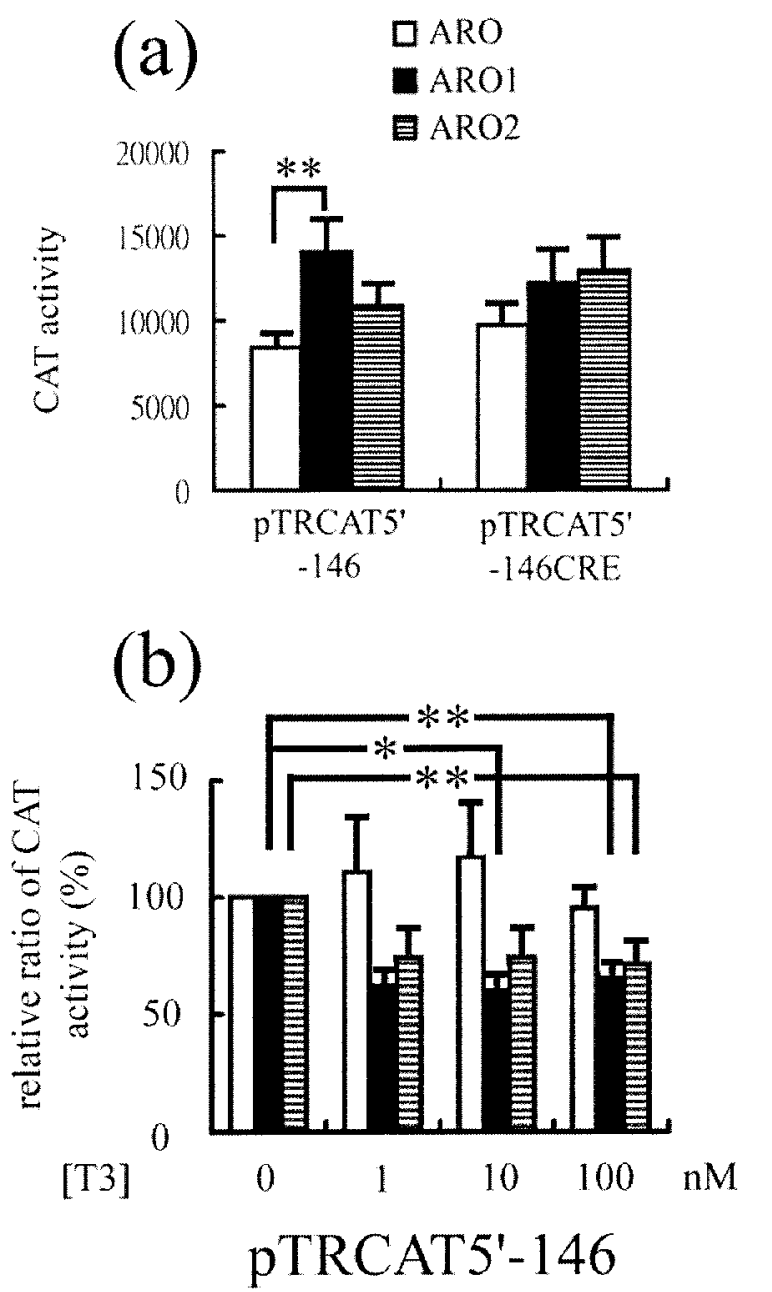

(c)

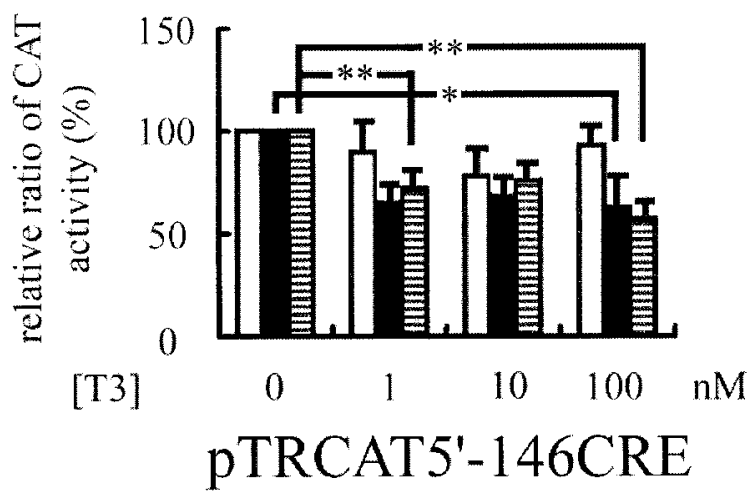

www.endocrinology.org sufficiently suppressed $\sim 34 \%$ to $\sim 40 \%$ and $\sim 26 \%$ to $\sim 29 \%$ of the basal activities of pTRCAT5' -146 in ARO1 and ARO2 cells respectively (Fig. $3 \mathrm{~b}$ ). The basal activity of the mutant reporter pTRCAT5'-146 CRE was equivalent to pTRCAT5'-146 in ARO1, ARO2 and the parental ARO cells (Fig. 2a). Again, T3 suppressed 32\% to $\sim 37 \%$ and $\sim 24 \%$ to $\sim 43 \%$ of the basal activities of pTRCAT5'-146 CRE in ARO1 and ARO2 cells respectively (Fig. 3c).

\section{T3 suppressed $p$ TRCAT5'-146 CRE activity in a $T R$-independent manner in ARO cells}

A point mutation of the minimal TSHR promoter did not lead to any change of the basal activity or the T3-induced inhibitory effect in ARO1 and ARO2 cells. Rather, in the TR $\beta 1$-deficient ARO cells, a T3-elicited suppression (nadir at $10 \mathrm{nM}$ to $22 \%$ ) of the pTRCAT5'-146 CRE activity was observed (Fig. 3c).

\section{Discussion}

The present study provided several lines of evidence to show that T3 down-regulates human TSHR gene expression, including: (i) The recombinant TR/RXR proteins bound the synthesized oligomer tshrp (Fig. 2a) that contained the flanking region of a consensus TRE half site existing in the minimal promoter region of the human TSHR gene (Fig. 1). (ii) In the absence of T3, the basal activity of a chimeric reporter pTRCAT $5^{\prime}-146$ that contains the fragment $(-146 /-9 \mathrm{bp})$ of the rat TSHR promoter was enhanced by TR $\beta 1$ overexpression in an anaplastic human thyroid cancer cell line, ARO (Fig. 3a). This finding is in agreement with our previous finding that TR $\beta 1$ overexpression up-regulated TSHR protein in ARO cells (Chen et al. 2000a). (iii) T3 inhibited the reporter activity in TR $\beta 1$-overexpressing transfectants,

Figure 3 T3 suppressed the CAT activity of reporter genes that contain the minimal TSHR promoter. (a) Values represent arbitrary units of the CAT activity detected from ARO, ARO1 and ARO2 cells. In the absence of T3, the reporter genes showed higher CAT activities in ARO1 and ARO2 cells than in ARO cells. A mutation of pTRCAT5'-146 (pTRCAT5'-146 CRE, see text) did not significantly influence the reporter activity. In (b) and (c), in the presence of the thyroid hormone, T3 (1, 10 and $100 \mathrm{nM})$ significantly suppressed the reporter gene activities in both ARO1 and ARO2 cells, as shown by the percentage, taking the basal CAT activity as $100 \%$. Thus (b) 40 and $29 \%$ of the basal activities of pTRCAT5'-146 were suppressed in ARO1 and ARO2 cells, but not in ARO cells. Similarly (c), T3 suppressed 37 and $43 \%$ of the basal activities of pTRCAT5'-146 CRE in ARO1 and ARO2 cells. In addition, T3 suppressed $22 \%$ of the basal activity of pTRCAT5'146 CRE in ARO cells. Values are means \pm S.E.M. of three independent experiments, each performed in duplicate. A difference was considered significant if the $P$ value was $<0.05$ by paired Student's $t$-test. ${ }^{*} P=0 \cdot 05 ;{ }^{*} P<0 \cdot 05$. 
but not in TR $\beta 1$-deficient parental cells (Fig. 3b). These findings fit in with the current concept of how TR reciprocally regulates the inherent negative regulatory elements of target genes; that is, the binding of the unliganded TR (TR/RXR) with corepressors recruits histone deacetylase (HDAC) and sequesters HDAC from the promoter, resulting in increased histone acetylation and transcriptional activation. In the presence of T3, the liganded TR separates from corepressors and binds to coactivators, which in turn makes HDAC available for the promoter and leads to target gene repression (Tagami et al. 1999).

In agreement with findings from footprint studies (Saiardi et al. 1994), we found that TR could only retard the oligomer tshrp when heterodimerized with RXR. In addition, when the oligomer F2 (which contained a positive TRE structure with inverted palindromic repeat) was applied as a control, tshrp showed a much weaker binding capability than F2 (Fig. 2). This finding is consistent with the results of Tagami et al. (1999), whose study suggested that nTRE could not bind TR as efficiently as positive TRE.

The restrictive expression of TSHR gene in the thyroid is determined by the limited expression of TTFs in the gland. However, ample expression of TSHR gene requires factors other than TTFs; for example, CRE-binding protein (CREBP) is needed to synergistically activate TSHR gene expression in conjunction with TTF1 (Saiardi et al. 1995). As a matter of fact, the octameric CRE-like motif of the rat minimal TSHR promoter has been recognized as a constitutive and/or inducible enhancer of the gene (Shimura et al. 1993). Interestingly enough, a TR $\alpha 1-$ mediated suppression of TSHR gene induced by TSH is also cAMP-dependent (Ikuyama et al. 1992, Saiardi et al. 1994, 1995). Whether this was secondary to an exclusive binding of TR to compete against CREBP binding with the overlapping responsive element remained unknown. As the overlapping binding sites for other classes of transcription factors have been specified as composite response elements (Hudson et al. 1990, Pearce \& Yamamoto 1993), we tried to verify whether a reconstructed consensus CRE motif would influence the binding of TR and transactivation of T3 to the overlapping TRE, which was mutated on the second nucleotide of the consensus hexameric (AGGTCA) structure that is considered important for DNA binding and is highly conserved among response elements recognized by all members of the TR/steroid hormone receptor superfamily (Glass 1994).

Unlike its higher basal activity detected in FRT cells, the creation of a consensus CRE structure by site-directed mutagenesis of pTRCAT5'-146 (Ikuyama et al. 1992) did not improve the basal CAT activity of pTRCAT5' -146 CRE in ARO cells with or without TR $\beta 1$ overexpression (Fig. 3a). This may be attributed to the defective expression of TTFs and/or impaired cAMP signal trans- duction pathway in ARO cells (Chen et al. 2000a). Furthermore, when the degree of T3-induced suppression was compared between the two reporters, we found that mutation of the -136 nucleotide that replaced the consensus TRE sequence in ACGTCA did not significantly influence the T3 effect in both TR $\beta 1$ overexpressing transfectants (Fig. $3 \mathrm{~b}$ and c). These findings indicate that TR did interact with the TRE-like sequence within pTRCAT5'-146 CRE even though we did not perform EMSAs to confirm DNA binding of TR/RXR proteins to the mutated sequence.

To our surprise, T3 suppressed the basal activity of pTRCAT5'-146 CRE in ARO cells, albeit to a lesser extent (Fig. 3c). Since ARO cells did not express TR proteins (Chen et al. 2000a), the T3-inducible suppression of pTRCAT5'-146 CRE activity in ARO cells was considered TR-independent. Although it remains unknown whether T3 modified a non-receptor factor such as CREBP that interacts with the composite regulatory element, the finding of reduced phosphorylated CREBP in toxic thyroid adenomas (Brunetti et al. 2000) suggests that maybe this is the case in our study. Actually, receptorindependent, non-genomic effects of T3 have been documented in a human squamous carcinoma cell line (Kesavan et al. 1991), an epidermoid carcinoma cell line (Ashizawa et al. 1991) and many others (Davis \& Davis 1996). It is suggested that the receptor-independent, non-genomic effects of T3 is carried out possibly through the activation of cytosolic protein kinases, such as protein kinase A, protein kinase C (Davis \& Davis 1996), and mitogen-activated protein kinase (Lin et al. 1999, Shih et al. 2001).

In conclusion, despite the lack of understanding of the sophisticated regulation of the composite regulatory element in the TSHR gene promoter, our observations may indicate a short-loop feedback control of TSHR gene expression by thyroid hormone in the thyroid.

\section{Acknowledgements}

The authors would like to thank Dr K S S Chang for his excellent comments and advice. We also would like to thank Dr S Y Cheng and Dr H Shimura for their generous provision of the plasmids. This work was supported by grants from Chang-Gung Memorial Hospital (CMRP918, CMRP1163) and the National Science Council of the Republic of China (NSC89-2314-B-182A-206).

\section{References}

Akiguchi I, Strauss K, Borges M, Silva JE \& Moses JE 1992 Thyroid hormone receptors and 3,5,3'-triiodothyronine biological effects in FRTL-5 thyroid follicular cells. Endocrinology 131 1279-1287.

Ashizawa K, McPhie P, Lin KH \& Cheng SY 1991 An in vitro novel mechanism of regulating the activity of pyruvate kinase M2 by thyroid hormone and fructose 1,6-bisphosphate. Biochemistry 30 7105-7111. 
Berlingieri MT, Akamizu T, Fusco A, Grieco M, Colletta G, Cirafici AM, Ikuyama S, Kohn LD \& Vecchio G 1990 Thyrotropin receptor gene expression in oncogene-transfected rat thyroid cells: correlation between transformation, loss of thyrotropin-dependent growth, and loss of thyrotropin receptor gene expression. Biochemical and Biophysical Research Communications 173 172-178.

Brabant G, Maenhaut C, Korle J, Scheumann G, Dralle H, Hoang-Vu C, Hesch RD, von zur Muhlen A, Vassart G \& Dumont JE 1991 Human thyrotropin receptor gene: expression in thyroid tumors and correlation to markers of thyroid differentiation and dedifferentiation. Molecular and Cellular Endocrinology 82 R7-R12.

Bronnegard M, Torring O, Boos J, Sylven C, Marcus C \& Wallin G 1994 Expression of thyrotropin receptor and thyroid hormone receptor messenger ribonucleic acid in normal, hyperplastic, and neoplastic human thyroid tissue. Journal of Clinical Endocrinology and Metabolism 79 384-389.

Brunetti A, Chiefari E, Filetti S \& Russo D 2000 The 3',5'-cyclic adenosine monophosphate response element binding protein (CREB) is functionally reduced in human toxic thyroid adenomas. Endocrinology 141 722-730.

Chatterjee VKK, Lee JK, Rentoumis A \& Jameson JL 1989 Negative regulation of the thyroid-stimulating hormone $\alpha$ gene by thyroid hormone: receptor interaction adjacent to the TATA box. PNAS 86 9114-9118.

Chen ST, Shieh HY, Lin JD, Chang KSS \& Lin KH 2000a Overexpression of thyroid hormone receptor $\beta 1$ is associated with TSHR gene expression and cell differentiation of an anaplastic thyroid cancer cell line. Journal of Endocrinology 165 379-389.

Chen WJ, Lin KH \& Lee YS $2000 b$ Molecular characterization of myocardial fibrosis during hypothyroidism: evidence for negative regulation of the pro-a1(I) collagen gene expression by thyroid hormone receptor. Molecular and Cellular Endocrinology 162 45-55.

Cheng SY 1995 New insights into the structure and function of the thyroid hormone receptor. Journal of Biomedical Science 2 77-89.

Civitareale D, Castelli MP, Falasca P \& Saiardi A 1993 Thyroid transcription factor 1 activates the promoter of the thyrotropin receptor gene. Molecular Endocrinology 7 1589-1595.

Cortell R \& Rawson RW 1944 The effect of thyroxine on the response of the thyroid gland to thyrotropic hormone. Endocrinology 35 488-498.

Davis PJ \& Davis FB 1996 Nongenomic actions of thyroid hormone. Thyroid 6 497-504.

Glass CK 1994 Differential recognition of target genes by nuclear receptor monomers, dimers, and heterodimers. Endocrine Reviews 15 391-407.

Harding PP \& Duester G 1992 Retinoic acid activation and thyroid hormone repression of the human alcohol dehydrogenase gene ADH3. Journal of Biological Chemistry 267 14145-14150.

Hudson LG, Santon JB, Glass CK \& Gill GN 1990 Ligand-activated thyroid hormone and retinoic acid receptors inhibit growth factor receptor promoter expression. Cell 62 1165-1175.

Ikuyama S, Shimura H, Hoeffler JP \& Kohn LD 1992 Role of the cyclic adenosine $3^{\prime}, 5^{\prime}$-monophosphate response element in efficient expression of the rat thyrotropin receptor promoter. Molecular Endocrinology 6 1701-1715.

Kesavan P, Mukhopadhayay S, Murphy S, Rengaraju M, Lazar MA \& Das M 1991 Thyroid hormone decreases the expression of epidermal growth factor receptor. Journal of Biological Chemistry 266 10282-10286.

Koenig RJ 1998 Thyroid hormone receptor coactivators and corepressors. Thyroid 8 703-713.

Kohn LD, Ban T, Okajima F, Shimura H, Shimura Y, Hidaka A, Giuliani C, Napolitano G, Kosugi S, Ikuyama S, Akamizu T, Tahara K \& Saji M 1995 Cloning and regulation of glycoprotein hormone receptor genes. In Molecular Endocrinology: Basic Concepts and Clinical Correlations, edn 1, pp 133-153. Ed. BD Weintraub. New York: Raven Press.

Lin HY, Davis FB, Gordinier JK, Martino LJ \& Davis PJ 1999 Thyroid hormone induces activation of mitogen-activated protein kinase in cultured cells. American Journal of Physiology 276 C1014-C1024.

Lin KH, Wu YH \& Chen SL 2001 Impaired interaction of mutant thyroid hormone receptors associated with human hepatocellular carcinoma with transcriptional coregulators. Endocrinology 142 653-662.

Moretti F, Farsetti A, Soddu S, Misiti S, Crescenzi M, Filetti S, Andreoli M, Sacchi A \& Pontecorvi A 1997 p53 re-expression inhibits proliferation and restores differentiation of human thyroid anaplastic carcinoma cells. Oncogene 14 729-740.

Pearce D \& Yamamoto KR 1993 Mineralocorticoid and glucocorticoid receptor activities distinguished by nonreceptor factors at a composite response element. Science 259 1161-1165.

Rapoport B \& Spaulding SW 1996 Mechanism of action of thyrotropin and other thyroid growth factors. In Werner and Ingbar's The Thyroid: a Fundamental and Clinical text, edn 7, pp 207-219. Eds LE Braverman \& RD Utiger. Philadelphia, PA: Lippincott-Raven.

Saiardi A, Falasca P \& Civitareale D 1994 The thyroid hormone inhibits the thyrotropin receptor promoter activity: evidence for a short loop regulation. Biochemical and Biophysical Research Communications 205 230-237.

Saiardi A, Falasca P \& Civitareale D 1995 Synergistic transcriptional activation of the thyrotropin receptor promoter by cyclic AMP-responsive-element-binding protein and thyroid transcription factor 1. Biochemical Journal 310 491-496.

Schuppert F, Deiters S, Rambusch E, Sierralta W, Dralle H \& Von Zur Muhlen A 1996 TSH-receptor expression and human thyroid disease: relation to clinical, endocrine, and molecular thyroid parameters. Thyroid 6 575-587.

Shih A, Lin HY, Davis FB \& Davis PJ 2001 Thyroid hormone promotes serine phosphorylation of $\mathrm{p} 53$ by mitogen-activated protein kinase. Biochemistry 40 2870-2878.

Shimura H, Ikuyama S, Shimura Y \& Kohn LD 1993 The cAMP response element in the rat thyrotropin receptor. Journal of Biological Chemistry 268 24125-24137.

Tagami T, Madison LD, Nagaya T \& Jameson L 1999 Nuclear receptor corepressors activate rather than suppress basal transcription of genes that are negatively regulated by thyroid hormone. Molecular and Cellular Biology 17 2642-2648.

Takasu N, Sato S, Tsukui T, Yamada T, Furihata R \& Makiuchi M 1974 Inhibitory action of thyroid hormone on the activation of adenyl cyclase-cyclic AMP system by thyroid-stimulating hormone in human thyroid tissues from euthyroid subjects and thyrotoxic patients. Journal of Clinical Endocrinology and Metabolism 39 772-778.

Williams GR \& Brent GA 1995 Thyroid hormone response elements. In Molecular Endocrinology: Basic Concepts and Clinical Correlations, edn 1, pp 217-239. Ed. BD Weintraub. New York: Raven Press.

Wood WM, Kao MY, Gordon DF \& Ridgway EC 1989 Thyroid hormone regulates the mouse thyrotropin $\beta$-subunit gene promoter in transfected primary thyrotropes. Journal of Biological Chemistry 264 14840-14847.

Yu S, Friedman Y, Richman R \& Burke G 1976 Altered thyroidal responsivity to thyrotropin induced by circulating thyroid hormones. A 'short-loop' regulatory mechanism? Journal of Clinical Investigation $\mathbf{5 7} 745-755$.

Received 13 March 2002

Accepted 4 July 2002 